\title{
Intelligent Image-Activated Cell Sorting and Beyond
}

\author{
Yasuyuki Ozeki ${ }^{1}$, Nao Nitta ${ }^{2,3}$, Takeaki Sugimura ${ }^{2,3}$, Akihiro Isozaki ${ }^{2}$, Hideharu Mikami', \\ Dino Di Carlo ${ }^{2,4,5,6}$, Yoichiroh Hosokawa ${ }^{7}$, Sotaro Uemura ${ }^{8}$, and Keisuke Goda ${ }^{2,3,9}$ \\ ${ }^{1}$ Department of Electrical Engineering and Information Systems, University of Tokyo, Tokyo 113-8656, Japan \\ ${ }^{2}$ Department of Chemistry, University of Tokyo, Tokyo 113-0033, Japan \\ ${ }_{3}^{3}$ Japan Science and Technology Agency, Saitama 332-0012, Japan \\ ${ }^{4}$ Department of Bioengineering, University of California, Los Angeles, Los Angeles, CA 90095, USA \\ ${ }^{5}$ Department of Mechanical Engineering, University of California, Los Angeles, Los Angeles, CA 90095, USA \\ ${ }^{6}$ California NanoSystems Institute, University of California, Los Angeles, Los Angeles, CA 90095, USA \\ ${ }^{7}$ Graduate School of Materials Science, Nara Institute of Science and Technology, Ikoma 630-0192, Japan \\ ${ }^{8}$ Department of Biological Sciences, University of Tokyo, Tokyo 113-0033, Japan \\ ${ }^{9}$ Department of Electrical Engineering, University of California, Los Angeles, Los Angeles, CA 90095, USA \\ Authore-mail address: ozeki@ee.t.u-tokyo.ac.jp
}

\begin{abstract}
We present a groundbreaking machine intelligence technology called "intelligent image-activated cell sorting" that achieves high-throughput image-triggered sorting of single cells by integrating high-speed fluorescence microscopy, cell focusing, cell sorting, and deep learning. $\odot 2019$ The Author(s)
\end{abstract}

OCIS codes: $110.0110,120.0120$.

\section{Introduction}

A fundamental challenge of biology is to understand the large heterogeneity of cells even with identical genomes [1]. The differences in composition, structure, and morphology of cells are strongly linked with their physiological functions such as proliferation, metabolism, secretion, differentiation, and signal transduction and an important aspect of cell identity. For example, cellular geometry such as size and shape influences intracellular signaling and regulates cellular growth and differentiation [2]. In addition, Wang et al. have found that the localization of $\mathrm{Ca}^{2+}$-binding proteins in chloroplasts varies with environmental $\mathrm{CO}_{2}$ conditions [3]. Furthermore, a range of other structural and morphological features such as nuclear shape, cytoskeleton structure, chromosome abnormality, chromatin structure, cellcell interaction, and RNA localization are likely linked to unique cellular functions [4].

To comprehend such cell-to-cell differences, a new technology is needed to rapidly search through and sort out cells with unique chemical and morphological features from large heterogeneous cell populations and then identify genes responsible for the features since conventional technologies have been limited in uncovering these relations. This is due to the trade-off between the volume and complexity of image data (which is correlated to accuracy) and the data transfer and image-processing speed (which is correlated to throughput). In fact, conventional high-throughput cell sorting technologies such as fluorescence-activated cell sorting (FACS) can only handle low-resolution data (i.e., $\sim 20$ light scattering and fluorescence signals without spatial information) for real-time data processing and sorting.

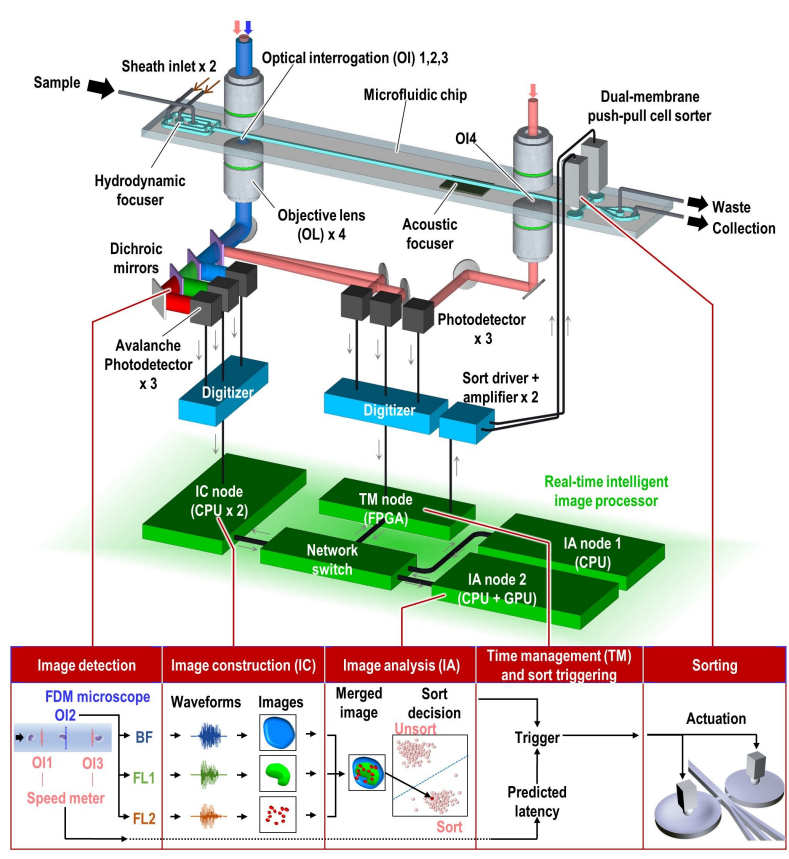

Fig. 1. Schematic of the intelligent image-activated cell sorter.
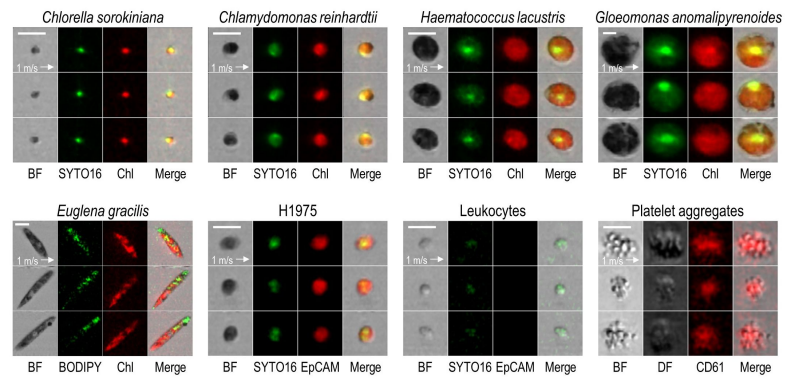

Fig. 2. Images of various types of cells captured by the intelligent image-activated cell sorter. 


\section{Results}

We developed an intelligent image-activated cell sorting (iIACS) technology to overcome this fundamental issue in cell biology [5]. As shown in Fig. 1, the iIACS integrates high-throughput cell microscopy [6], microfluidic cell focusing, and microfluidic cell sorting [7] on a hybrid software-hardware management infrastructure that runs a telecom-grade 10-Gbps all-Internet-Protocol (IP) architecture. It employs a radically different data-management infrastructure and hence realizes real-time image-based intelligent cell search and sorting at an unprecedented throughput of about 100 events per second. This multidisciplinary system enables high flexibility, high scalability, and automated operation for data acquisition, data processing, decision-making, and actuation, all within $32 \mathrm{~ms}$ even with deep-learning algorithms. In a sense, the iIACS is an imaging version of FACS, which offers many more capabilities in both technological and applicational aspects (See the operation of the intelligent image-activated cell sorter: https://youtu.be/7cuXRQgRvWI).

The functionality of the intelligent image-activated cell sorter is as follows. Suspended cells in a sample tube injected into the intelligent image-activated cell sorter are focused by the hydrodynamic focuser into a single stream in a microchannel on a chip, imaged by the frequency-division-multiplexed fluorescence microscope, analyzed by the real-time intelligent image processor, and sorted by the dual-membrane push-pull cell sorter triggered by decisions made by the image processor. The entire process is operated in a fully automated and real-time manner (See the functionality of the intelligent image-activated cell sorter: https://youtu.be/1SYgtBZwKYA).

We evaluated the imaging capability of the iIACS with diverse cell types. Fig. 2 shows bright-field and twocolor fluorescence images of various microalgal and human cells in a high-speed flow at a speed of $1 \mathrm{~m} / \mathrm{s}$. The high quality of the images indicates that the iIACS is capable of identifying the intracellular molecular distribution and morphological features of various cell types.

To show the broad applicability of the iIACS, we applied it to high-content sorting of microalgal and blood cells based on protein localization and cell-cell interaction, respectively. In the first application, we used the technology to sort Chlamydomonas reinhardtii mutants with aberrantly distributed low- $\mathrm{CO}_{2}$ inducible protein $\mathrm{B}$ under low- $\mathrm{CO}_{2}$ conditions [Fig. 3(a)]. The technology identified 221,947 events and sorted 2,021 with the aberrant protein localization (less than $1 \%$ of the total population). This sorting process would normally take $\sim 6$ months if it was done manually, but the technology conducted it in less than 40 minutes. In the second application, we used the technology to sort platelet aggregates from human blood [Fig. 3(b)]. Our convolutional neural network calculated the probability of identifying an object in each bright-field image as a leukocyte, single platelet, or platelet aggregate in fixed and hemolysed human blood, showing the ability of the convolutional neural network to differentiate a single platelet and a doublet, which is not possible with FACS. Applying progressive gating to the probabilities yielded high specificity and sensitivity of $99 \%$ and $82 \%$, respectively, in detecting platelet aggregates, and a high purity of $79.5 \%$ in sorting platelet aggregates. This sorting process would normally take $\sim 1$ day if it was done manually, but the technology performed it in less than 1 minute.

(a)
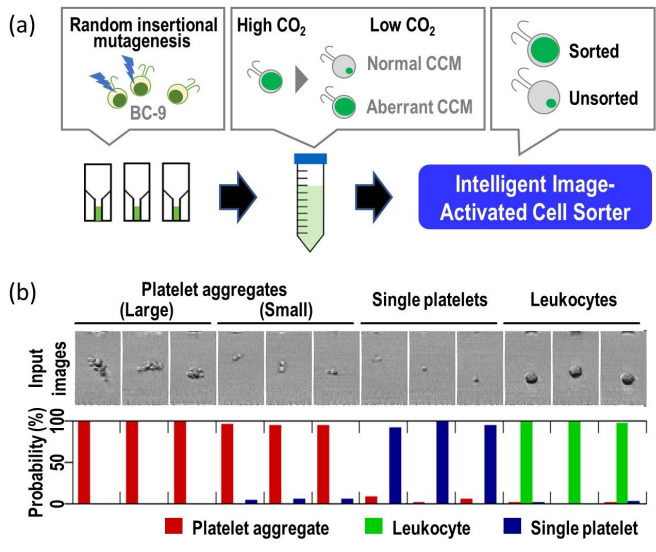
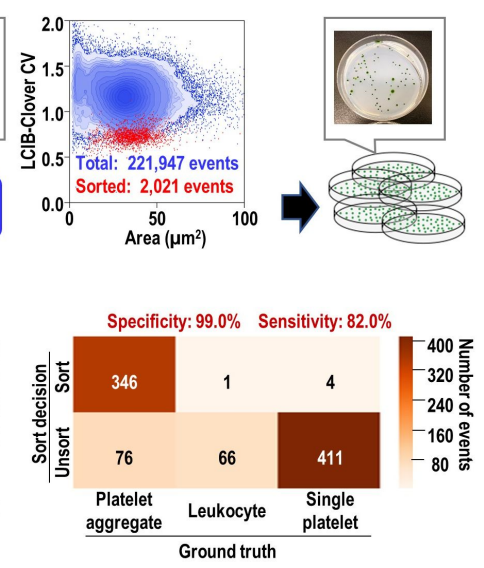

Fig. 3. High-content analysis and sorting of (a) microalgal cells and (b) blood cells.

\section{References}

[1] S. J. Altschuler et al., Cell 141, 559 (2010).

[2] T. von Erlach et al., Nat. Materials 17, 237 (2018).

[3] L. Wang et al., PNAS 113, 12586 (2016).

[4] A. E. Moor et al., Science 357, 1299 (2017).
[5] N. Nitta et al., Cell 175, 266 (2018).

[6] H. Mikami et al., Optica 5, 117 (2018).

[7] S. Sakuma et al., Lab Chip 17, 2760 (2017). 